\title{
Sismicidade local e mecanismo focal em Correntina - BA
}
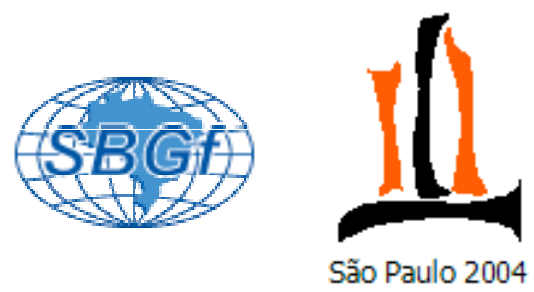

Afonso E. V. Lopes, Marcelo Assumpção e José Roberto Barbosa, IAG-USP, Brasil

Copyright 2004, SBGf - Sociedade Brasileira de Geofísica

Este texto foi preparado para a apresentação no I Simpósio de Geofísica da Sociedade Brasileira de Geofísica, São Paulo, 26-28 de setembro de 2004. Seu conteúdo foi revisado pela Comissão Tecno-científica do I SR-SBGf mas não necessariamente representa a opinião da SBGf ou de seus associados. E proibida a reprodução total ou parcial deste material para propósitos comerciais sem prévia autorização da SBGf.

\section{Resumo}

Em julho de 2003 foram sentidos tremores no município de Correntina, BA. Alguns meses depois são instaladas quatro estações sismográficas na região epicentral, e são registrados mais de cem eventos com magnitudes $\left(m_{D}\right)$ entre 0,2 e 1,5, vários deles com ondas de superfície bem nítidas. Com dados de polaridade das ondas $\mathrm{P}$ e $\mathrm{S}$, e modelagem das ondas de superfície obteve-se um modelo de velocidades para a porção mais rasa da crosta superior, e determinou-se um mecanismo focal para a atividade. Este mecanismo focal preliminar indica falhamento inverso com direção de compressão E-W. A profundidade focal é da ordem de $1 \mathrm{~km}$.

\section{Introdução}

Em agosto de 2003 o IAG-USP iniciou a instalação de uma série de estações sismográficas no interior do Cráton do São Francisco para o aumento da área de estudos com tomografia sísmica. Dentro desse projeto, instalou-se uma estação sismográfica na cidade de Correntina-BA (Figura 1).

$\mathrm{Na}$ atualização do Boletim Sísmico Brasileiro constatouse a ocorrência de três sismos na região de Correntina, todos com magnitudes próximas de 3.0 , em julho de 2003 , e registrados por estações a até $630 \mathrm{~km}$ do epicentro. A sismicidade da região foi confirmada com a primeira coleta de dados da estação sismográfica de banda larga CRTB. A partir dai o grupo de sismologia concentrou esforços na instalação de outras três estações sismográficas de período curto na região.

A sismicidade de Correntina era desconhecida até então (Berrocal et al. 1984 - Figura 1), e em paralelo ao seu estudo, obteve-se um modelo para a estrutura da parte mais rasa da crosta superior (primeiro $1 \mathrm{~km}$ ). $\mathrm{O}$ mecanismo focal dos sismos foi determinado com a metodologia citada em Lopes e Assumpção (2004).

As estações sismográficas foram posicionadas a partir de informações da população local obtidas em um levantamento macrossísmico, mas infelizmente o arranjo não ficou com a melhor geometria. Em um primeiro contato com a população sentimos preocupação quanto aos "estrondos" (sismos) que ocorriam com bastante freqüência. $\mathrm{Na}$ Fazenda Patos, foi sentido uma espécie de "rolo compressor" passando por baixo dos pés logo após os "estrondos", referindo-se provavelmente as ondas Rayleigh observadas nos sismogramas. Moradores da Fazenda
Tapicuru, disseram ter ouvido o "estrondo" enquanto assistiam TV.

Pessoas próximas ao epicentro fizeram relatos interessantes, indicando intensidades de III a IV. O Sr Carlindo, da Fazenda Conceição, afirmou que a atividade havia cessado, mas que no ano anterior (07/2003) houve um "terremoto" que chegou a balançar a geladeira do vizinho. Na mesma fazenda, o sr Otacilio disse que as prateleiras chegaram a balançar em sua casa. O sr João Rego, da Fazenda Harmonia, disse ter sentindo os sismos de julho que chacoalharam prateleiras e telhas.

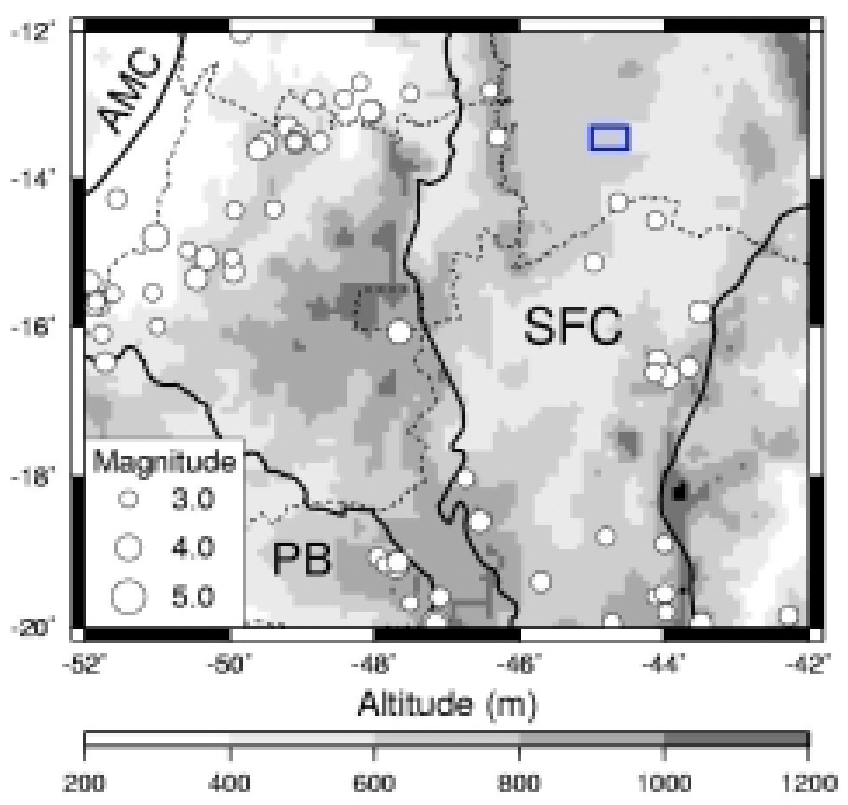

Figura 1 - O quadrado azul indica a posição da cidade de Correntina, linhas continuas os limites das principais províncias geológicas (Cráton do São Francisco, SFC; Cráton do Amazonas, AMC; e Bacia do Paraná, PB), linhas tracejadas limites políticos, e os círculos brancos representam os maiores sismos conhecidos no território brasileiro.

Todos os relatos nas proximidades dos epicentros indicavam que a atividade sísmica havia cessado, enquanto que na região onde as estações sismográficas foram instaladas os relatos não eram tão dramáticos, mas a população ouvia os "estrondos" quase diáriamente.

Junto ao levantamento macrossísmico na região (Figura 2a), foram feitos esclarecimentos sobre os "estrondos" que tanto amedrontavam a população local (Figura 2b). Muitas pessoas faziam analogias com o fim do mundo, e ficaram mais tranqüilas após um breve informe sobre a origem dos "estrondos". 

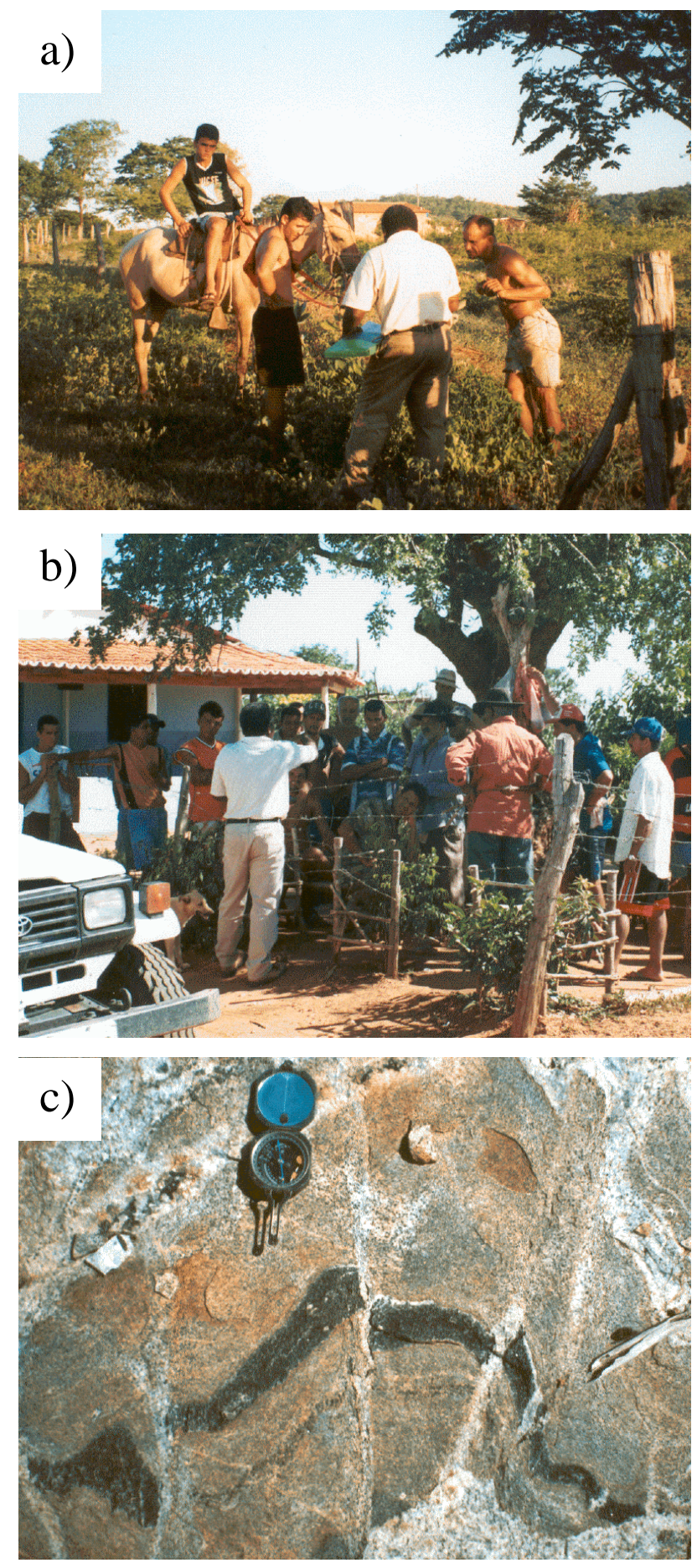

Figura 2 - Fotos do levantamento macrossísmico. Em a) enquanto o técnico se informava sobre possíveis pontos de afloramento com a população, um aluno fazia uma medida com GPS para localização no mapa topográfico. Prestou-se esclarecimento à população local, e no açougue (b) conseguimos informações sobre o ponto onde instalamos uma das estações sismográficas. Em toda a região observam-se estruturas como a apresentada em c).

\section{Sísmicidade da Região}

O mapa de sismicidade da área de estudo é apresentado na Figura 3 junto com dados de topografia (SRTM, NASA), e é limitado na Figura 1 pelo retângulo azul. A sismicidade se concentra nas margens do rio Arrojado (Figura 3), mas também registrou-se um outro foco com dois sismos próximos ao Rio Corrente (35 km a NW das estações).

Durante o levantamento macrossísmico um morador da Fazenda Patos, disse que em um dos "estrondos" a janela chegou a bater, o fio da antena tremeu, e que na casa de seu vizinho (500 m ao lado) xícaras caíram. Disse ainda que sentiu a vibração passando sob seus pés, apontou uma direção, e disse que os "estrondos" vinham da Serra ao lado de sua casa. A Figura 3 mostra que realmente os sismos estão próximos a um alto topográfico.

Os sismos registrados por apenas uma estação sismográfica tiveram seu epicentro determinado com o movimento de partícula na horizontal (Lopes et al. 2003), e os registrados por três ou mais estações com o programa HYPO71 (Lee \& Lahr 1978).

O modelo de velocidade utilizado nas determinações hipocentrais satisfaz a curva de dispersão da velocidade de grupo das ondas Rayleigh, e é composto de duas camadas e um semi-espaço. A primeira e a segunda camada têm espessuras de $0.3 \mathrm{~km}$ e $0.7 \mathrm{~km}$, e velocidades da onda $P$ de $4.3 \mathrm{~km} / \mathrm{s}$ e $5.2 \mathrm{~km} / \mathrm{s}$, respectivamente. A velocidade no semi-espaço é de $6.1 \mathrm{~km} / \mathrm{s}$.

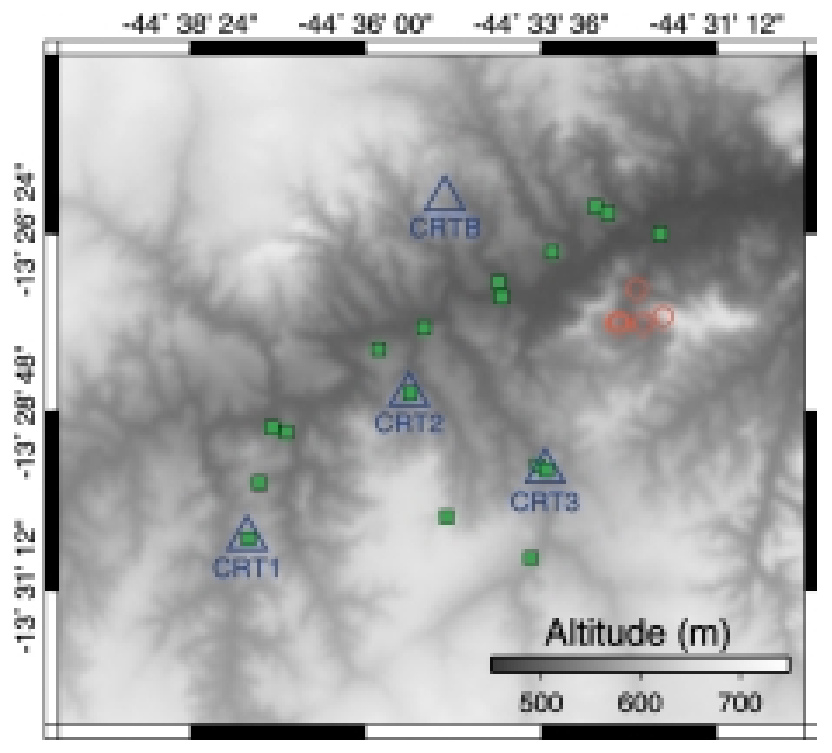

Figura 3 - Mapa topográfico (SRTM) e sismicidade da região de Correntina. Os triângulos azuis são estações sismográficas, círculos vermelhos os epicentros (apenas eventos registrados por quatro estações), e quadrados verdes são os pontos onde se fez o levantamento macrossísmico. A leitura dos tempos de chegada das ondas $P$ e $S$ foram feitas correlacionando-se a forma de onda de todos os eventos, garantindo boas posições relativas. 


\section{Mecanismo Focal}

Acredita-se que a fonte de todos os sismos é a mesma, já que esses se concentram em uma área bastante pequena e as polaridades observadas em cada estação são geralmente consistentes. Desse modo, pode-se utilizar dados de vários sismos na determinação de um único mecanismo focal.

Seis eventos registrados pelas quatro estações sismográficas foram utilizados na determinação de um mecanismo focal composto, utilizando os dados de polaridade das ondas $\mathrm{P}, \mathrm{SH}$, a razão de amplitude $\mathrm{SH} / \mathrm{P}$ (Figura 4), e modelagem das ondas Rayleigh (Figura 5).

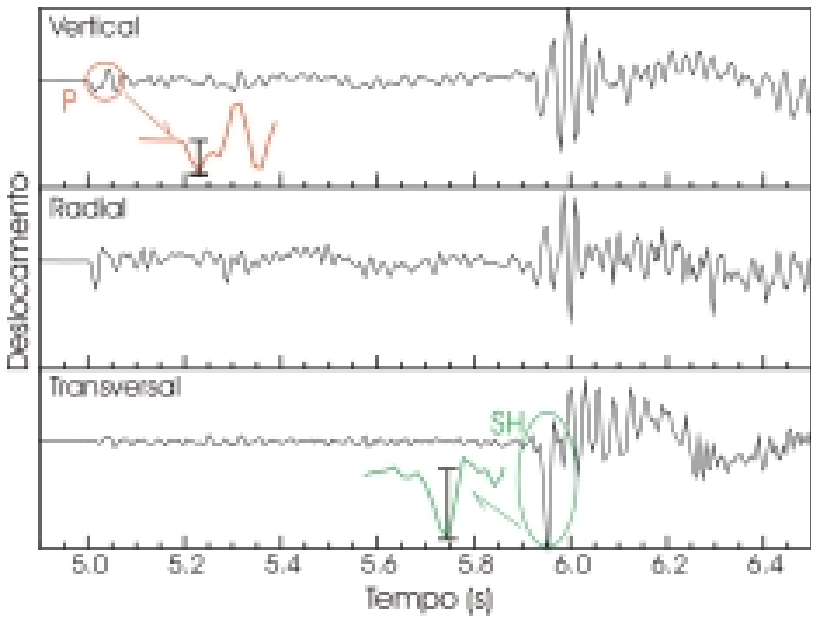

Figura 4 - Componente vertical, radial e transversal de um sismo (26/03/04) registrado em CRT2. As escalas verticais estão normalizadas, e as ampliações ilustram as amplitudes utilizadas no cálculo da razão $S H / P$. Note que a polaridade da $P$ é para baixo, e a da SH é esquerda.

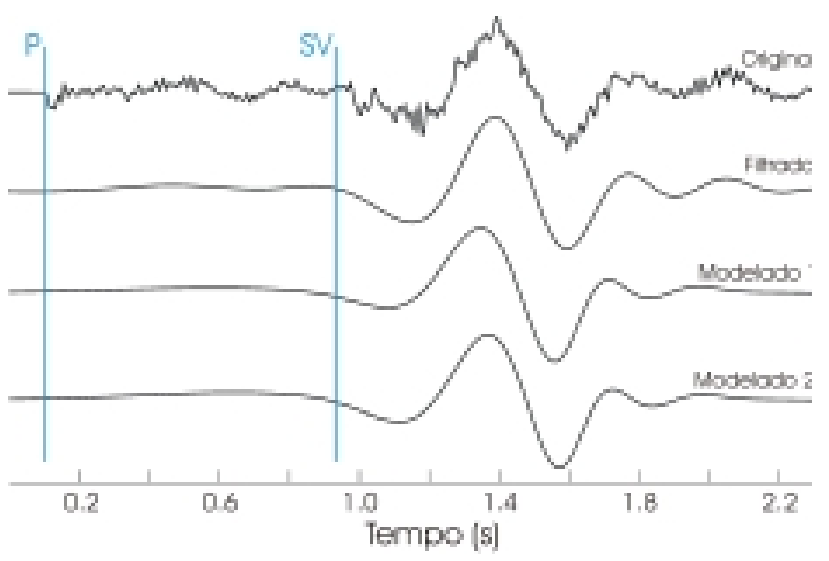

Figura 5 - O primeiro traço é o sismograma original (em deslocamento). Este sinal é filtrado com um filtro match para isolar o modo fundamental da onda Rayleigh (segundo traço). Os dois últimos traços correspondem aos sismogramas sintéticos gerados com os dois melhores mecanismos focais (Fig. 6b) determinados com a metodologia descrita em Lopes e Assumpção (2004).
A polaridade das ondas $\mathrm{P}$ e $\mathrm{SH}$, e a razão $\mathrm{SH} / \mathrm{P}$ foram utilizadas na seleção de uma população de mecanismos focais com o programa FocMec (Snoke et al., 1984). As soluções que melhor satisfazem estes dados estão na Fig. 6a. A modelagem das ondas Rayleigh foi feita com a metodologia descrita em Lopes e Assumpção (2004), usando um pacote de análise sismológica de Herrmann e Ammon (2002), para avaliar a população inicial (Figura 6a). As duas soluções que melhor ajustam as ondas Rayleigh estão indicadas na Fig. 6b.

Os dois melhores mecanismos focais apresentam um bom ajuste para a forma de onda (Figura 5), e correspondem as soluções (strike, dip, rake): $\left(10^{\circ}, 40^{\circ}, 90^{\circ}\right)$ e $\left(340^{\circ}, 45^{\circ}, 60^{\circ}\right)$. Ambas as soluções representam falhas inversas com direção de compressão E-W.
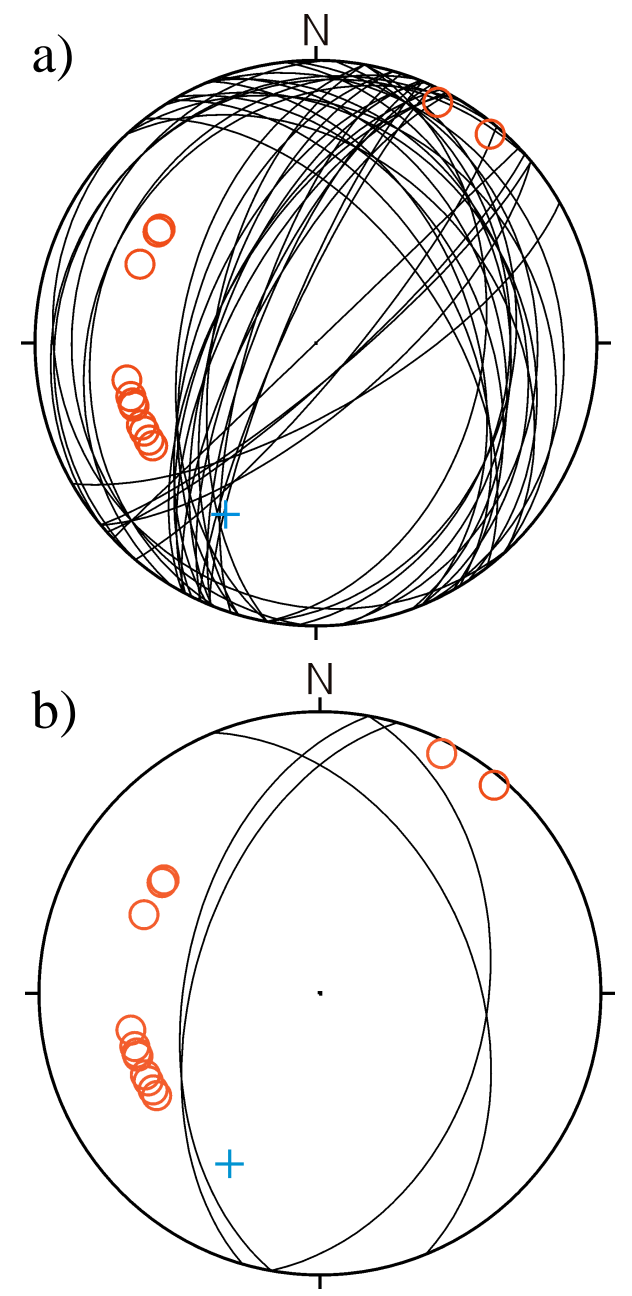

Figura 6 - Soluções preliminares para o mecanismo focal dos sismos de Correntina. As bolas vermelhas representam polaridades negativas para a onda $P$, e a cruz azul polaridade positiva. a) Todos os mecanismos focais que satisfazem as polaridades das ondas $P$ e $S H$, e razão $S H / P$. b) Os dois melhores mecanismos focais (segundo a modelagem das ondas Rayleigh mostrado na Fig. 5). 


\section{Considerações Finais}

Grande parte dos dados obtidos em Correntina ainda não foram processados, e possivelmente acrescentarão informações que poderão dar mais credibilidade ao Mecanismo focal determinado.

A metodologia utilizada na modelagem com as ondas de superfície se mostrou bastante eficiente, mas há necessidade de um estudo mais detalhado sobre a relação entre os parâmetros de atenuação $\left(Q_{p}\right.$ e $\left.Q_{s}\right)$ e a profundidade focal do evento. Maior atenuação inelástica (menor Q) diminui as freqüências mais altas das ondas Rayleigh. O mesmo efeito ocorre aumentando-se a profundidade focal. Esta interdependência pode causar incertezas no mecanismo focal.

A profundidade focal é muito sensível ao modelo de velocidade, podendo variar de $2 \mathrm{~km}$ para um modelo de semi-espaço, até $0.3 \mathrm{~km}$ para o modelo determinado com a dispersão das ondas Rayleigh. Neste caso, a modelagem das ondas de superfície pode ser importante na determinação da profundidade focal.

Em uma segunda etapa deste trabalho serão estudados os outros dois sismos que ocorreram fora da área apresentada na Figura 3.

\section{Agradecimentos}

Ao Observatório Sismológico da Universidade de Brasília (SIS) pelo apoio em trabalho de campo. Em especial ao prof $^{\circ}$. Lucas Barros, pelo empréstimo de um carro e motorista para uma manutenção de emergência nas estações sismográficas de Correntina, e o técnico Marcos pela ajuda na manutenção das estações sismográficas nesta viagem. A prof ${ }^{a}$. Naomi Ussami pelos esclarecimentos sobre os dados geofísicos da região, e dados bibliográficos. Desejamos expressar aqui nosso agradecimento à FAPESP pelo financiamento de nossas pésquisas (processo 03/12204-8) e desenvolvimento da ciência no país.

\section{Referências}

Berrocal, J., Assumpção, M., Antezana, R. et al., 1984. Sismicidade do Brasil, IAG/USP - CNEN, São Paulo, Brasil.

Herrmann, R.B. \& Ammon, C.J., 2002. Computer Programs in Seismology: Surface Waves, Receiver Functions and Crustal Structure, Saint Louis University.

Herrmann, R.B., 2001. Computer Programs in Seismology: Surface Waves, Receiver Functions and Crustal Structure, Saint Louis University.

Lee, W.H.K. \& Lahr, J.C. (1978) HYPO71 (revised): A Computer Program for Determining Hypocenter, Magnitude and First Motion Pattern of Local Earthquakes, USGS Open File Report 75-311.
Lopes, A.E.V \& Assumpção, M., 2004. Mecanismo focal de sismos próximos com polaridades de ondas $\mathrm{P}$ e modelagem de ondas de superfície: Aplicação ao estudo do sismo de Brasília-DF (20/11/2000), submetido ao I Simpósio Regional da SBGf.

Lopes, A.E.V., Assumpção, M. \& Barbosa, J.R., 2003. Prática de Determinação Hipocentral, com introdução ao uso do SAC, Apostila do curso de férias "Introdução à Sismologia", IAG-USP.

Snoke, J.A., J.W. Munsey, A.C. Teague \& G.A. Bollinger, 1984. A program for focal mechanism determination by combined use of polarity and SV-P amplitude ratio data, Earthquake Notes, 55. 